\title{
Reflets
}

Revue d'intervention sociale et communautaire

\section{Construction de la masculinité : de jeunes Franco-manitobains s'expriment}

\section{Stéfan Delaquis}

Volume 21, numéro 2, automne 2015

$20^{\mathrm{e}}$ anniversaire... déjà !

URI : https://id.erudit.org/iderudit/1035437ar

DOI : https://doi.org/10.7202/1035437ar

Aller au sommaire du numéro

Éditeur(s)

Reflets, Revue d'intervention sociale et communautaire

ISSN

1203-4576 (imprimé)

1712-8498 (numérique)

Découvrir la revue

Citer cet article

Delaquis, S. (2015). Construction de la masculinité : de jeunes

Franco-manitobains s'expriment. Reflets, 21(2), 161-193.

https://doi.org/10.7202/1035437ar
Résumé de l'article

Cet article s'intéresse à la construction de la masculinité des garçons en contexte scolaire. Plus particulièrement, il vise des garçons francophones du Manitoba de la onzième et de la douzième année et veut mieux cerner le phénomène de la construction de la masculinité des garçons à travers leur participation à des groupes sociaux et à des communautés de pratique à l'école. Les garçons négocient et présentent diverses versions de la masculinité dans différentes situations sociales et chaque contexte social a un impact considérable sur l'élaboration de leur masculinité, ce qui engendre des effets sur leur vécu scolaire. La présente étude est qualitative de type ethnographique et les données ont été analysées à partir de la théorie sociale du genre de Connell $(1987 ; 1995 ; 2002)$ en portant un regard particulier sur la masculinité hégémonique, ainsi que de celle des communautés de pratique développée par Wenger (2005). Les résultats indiquent que la masculinité présente dans ce milieu semble être fondée sur un modèle traditionnel et comprend une adhésion à une construction de la masculinité hégémonique. Enfin, l'image de la masculinité ne semble pas permettre aux garçons de contempler d'autres types de masculinité.
Tous droits réservés @ Reflets, Revue d’intervention sociale et communautaire, 2015
Ce document est protégé par la loi sur le droit d'auteur. L'utilisation des services d'Érudit (y compris la reproduction) est assujettie à sa politique d'utilisation que vous pouvez consulter en ligne.

https://apropos.erudit.org/fr/usagers/politique-dutilisation/ 


\section{Construction de la masculinité : de jeunes Franco-manitobains s'expriment}

Stéfan Delaquis, Ph. D.

Faculté d'éducation et des études professionnelles, Université de Saint-Boniface

\section{Résumé}

Cet article s'intéresse à la construction de la masculinité des garçons en contexte scolaire. Plus particulièrement, il vise des garçons francophones du Manitoba de la onzième et de la douzième année et veut mieux cerner le phénomène de la construction de la masculinité des garçons à travers leur participation à des groupes sociaux et à des communautés de pratique à l'école. Les garçons négocient et présentent diverses versions de la masculinité dans différentes situations sociales et chaque contexte social a un impact considérable sur l'élaboration de leur masculinité, ce qui engendre des effets sur leur vécu scolaire. La présente étude est qualitative de type ethnographique et les données ont été analysées à partir de la théorie sociale du genre de Connell $(1987 ; 1995 ; 2002)$ en portant un regard particulier sur la masculinité hégémonique, ainsi que de celle des communautés de pratique développée parWenger (2005). Les résultats indiquent que la masculinité présente dans ce milieu semble être fondée sur un modèle traditionnel et comprend une adhésion à une construction de la masculinité hégémonique. Enfin, l'image de la masculinité ne semble pas permettre aux garçons de contempler d'autres types de masculinité.

Mots clés : construction de la masculinité, groupes sociaux, théorie des communautés de pratique, théorie sociale du genre, masculinité hégémonique 


\section{Abstract}

\section{Introduction}

«Bon nombre

de parents et d'enseignantes ou d'enseignants se préoccupent de cette question complexe qui prend de plus en plus de place dans l'arène publique..."
This article seeks to facilitate an understanding of how boys construct their masculinity in a school environment. More specifically, Franco-Manitoban boys from grades 11 and 12 were interviewed in order to better understand this phenomenon through their participation in social groups and communities of practice at school. Boys negotiate and display multiple versions of masculinity in diverse social circumstances. Each social context has a significant impact on constructing masculinity that in turn affects boys' lives in school. This thesis is qualitative in nature and uses an ethnographic "lens". Connell's $(1987 ; 1995 ; 2002)$ social theory of gender with a particular look at hegemonic masculinity, as well as Wenger's (2005) social theory of communities of practice, were used to analyse data. Results indicate that the masculinity in this particular school environment seems to be based on a traditional model, which includes an adherence to a hegemonic masculinity. Furthermore, the image that relates to the ideal masculinity does not allow boys to consider other forms thereof.

Key words: construct of masculinity, social groups, social theory of gender, social theory of communities of practice, hegemonic masculinity
Le champ d'études portant sur la réalité du jeune adolescent à l'école a pris de l'ampleur au Canada, en Australie, au RoyaumeUni et aux États-Unis au cours des dernières années. Plus particulièrement, il semble y avoir une résurgence importante de l'intérêt envers le développement social de la masculinité des garçons et de son lien avec l'éducation (Connell, 2008; Frosh, Phoenix et Pattman, 2002; Frank, et collab., 2003; Kehler, 2004; Swain, 2006). Bon nombre de parents et d'enseignantes ou d'enseignants se préoccupent de cette question complexe qui prend de plus en plus de place dans l'arène publique (Connell, 
2008; Frank, et collab., 2003; Kehler, 2004). Les hypothèses sont nombreuses, mais parfois peu approfondies. Selon Frank et collab. (2003) et Kehler (2004), on accorde peu d'attention à la complexité ainsi qu'à la diversité de la masculinité et à la façon dont celle-ci est négociée par les adolescents en milieu scolaire. À première vue, il semble que ceux-ci présentent diverses versions de la masculinité dans leurs différentes situations sociales, telles que leur famille, leur communauté, leur école et leurs loisirs. Chacun de ces contextes accorde aux adolescents l'occasion d'assembler des idées, d'affirmer des valeurs et d'adopter des comportements qui contribuent à la construction de leur masculinité (Swain, 2005). Cependant, l'identité masculine semble se construire graduellement à travers la participation aux activités de groupes sociaux. Ces groupes sociaux font partie du vécu scolaire et ils forment le noyau à partir duquel les adolescents négocient leur masculinité.

\section{Classification des divers types de masculinités}

Peu importe la perspective adoptée, psychologique, anthropologique, sociologique, la plupart des chercheurs s'entendent sur le fait que la masculinité ne constitue pas une notion monolithique ou unidimensionnelle. On le constate aisément autour de nous et ailleurs dans le monde en ce tournant du $\mathrm{XXI}^{\mathrm{e}}$ siècle, la masculinité adopte plusieurs formes. Qu'elles soient plus ou moins admises socialement selon leur expression est une autre histoire, largement documentée par la recherche sur la conformité sociale, l'identité et bien d'autres phénomènes connexes.

L'expression "masculinité hégémonique " a été proposée par les chercheurs Carrigan, Connell et Lee (1985) et définie davantage par Connell (1987; 1995; 1996; 2000). Ce dernier donne un aperçu de la façon dont le pouvoir est incorporé au développement de la masculinité. Connell (2000) maintient que dans divers milieux il y a une hiérarchie des masculinités et une ascendance par laquelle une forme de masculinité domine les 
"...plusieurs garçons croient qu'ils doivent adhérer aux normes et aux valeurs établies par la masculinité hégémonique afin d'adopter des comportements qui leur permettent de démontrer leur virilité." "

\section{"...la masculinité} hégémonique est une configuration de pratiques et de valeurs liées au genre d'une personne qui assure une forme de domination de la part du sexe masculin..." autres. Selon Connell (1990), Gilbert et Gilbert (1998) et Wetherell et Edley (1999), la masculinité hégémonique est souvent celle qui est idéalisée par bon nombre de garçons et d'hommes. Elle empiète d'ailleurs sur les diverses façons dont ces derniers peuvent interpréter la masculinité. Pour leur part, Kenway et Fitzclarence (1997), Mills (2001) et Beasly (2008) soutiennent que cette forme de masculinité est à la fois traditionnelle et habituelle, et que plusieurs garçons croient qu'ils doivent adhérer aux normes et aux valeurs établies par la masculinité hégémonique afin d'adopter des comportements qui leur permettent de démontrer leur virilité. Ces comportements sont souvent appris au moyen d'interactions avec autrui et de fréquentations dans le cadre de différentes activités sociales. Cela étant dit, les comportements liés à la masculinité hégémonique peuvent inclure la dévalorisation du genre féminin (Mankowski, et Maton, 2010), une surutilisation de jurons ou le fait de poser des gestes sexuels inappropriés tels que se coller le corps très près d'une femme, faire des attouchements sur les fesses ou les cuisses d'une femme ou faire des commentaires sur l'attrait physique ou le corps d'une femme. Par ailleurs, Swain (2006) affirme que la masculinité hégémonique présente dans un milieu est déterminée par les caractéristiques observables de la culture ambiante telles que la conformité ou la résistance et la passivité ou la violence en relation avec les formes d'autorité présentes dans le contexte. Par exemple, selon Adler, Kless et Adler (1992), les comportements dits " machos » comprennent souvent la belligérance et l'affrontement. Les plus haut placés dans ce réseau des relations sociales sont souvent des fauteurs de trouble qui cherchent à attirer constamment l'attention des autres par leurs interactions agressives avec l'autorité. C'est donc de cette façon qu'ils accèdent à un statut élevé, bénéficient d'un prestige et servent des modèles susceptibles d'avoir une portée négative auprès de leurs pairs. Tout bien considéré, la masculinité hégémonique est une configuration de pratiques et de valeurs liées au genre d'une personne qui assure une forme de domination de la part du sexe masculin (Connell, 1995). En outre, cette forme de masculinité serait une réaction négative exagérée à des idéologies et à des comportements dits féminins (Connell, et Messerschmidt, 2005). 
Pour Wetherell et Edley (1999), la masculinité hégémonique n'est pas un trait de personnalité ou un caractère particulier, mais plutôt un ensemble de normes et de règles symboliques associées à diverses activités. Cette forme de masculinité fait appel à des caractéristiques socioculturelles telles que l'hétérosexualité, l'habileté athlétique, la force physique, le courage, la condition physique, la compétitivité, l'autonomie et le contrôle des émotions (Levant, et collab., 2007). Plusieurs modèles de masculinité hégémonique sont liés au corps masculin. Ils se manifestent par des bagarres dans les couloirs de l'école, des conduites agressives lors de compétitions sportives ou des situations narcissiques où, par exemple, les jeunes hommes prennent des poses devant le miroir de la salle d'haltérophilie. Les garçons apprennent vite en observant les autres garçons qu'un corps d'athlète bien proportionné est en général désiré et valorisé par la plupart d'entre eux, car il transmet un message de virilité, de puissance, de robustesse et de pouvoir qu'on attribue dans plusieurs contextes plus fréquemment à l'homme (Gilbert, et Gilbert, 1998). De plus, il semble que l'image d'une identité sexuelle masculine repose souvent sur la notion de "performance » (Gilbert, et Gilbert, 1998). Par conséquent,

"...les pratiques et les comportements sociaux qu'entretiennent les garçons et les hommes dans le but d'affirmer leur masculinité sont souvent liés à ce qu'ils font avec leur corps. " les pratiques et les comportements sociaux qu'entretiennent les garçons et les hommes dans le but d'affirmer leur masculinité sont souvent liés à ce qu'ils font avec leur corps. Dans certains milieux, le garçon qui développe ses muscles par l'exercice et l'entraînement ou celui qui donne de bonnes mises en échec au hockey sera davantage apprécié par ses amis et ses pairs.

Connell (1995) et Swain (2006) distinguent d'autres formes de masculinité en plus de celle de nature hégémonique. En effet, la masculinité dite "complice " se caractérise comme étant une forme aspirante de la masculinité hégémonique. Le regroupement de garçons qui correspond au profil complice se trouve fréquemment à la périphérie du groupe dominant. Ils sont à l'écart en raison d'une insuffisance de traits ou d'habiletés de la forme masculine idéalisée. Quoique certains d'entre eux fassent preuve d'habiletés athlétiques et affichent différentes qualités telles que la persévérance et le contrôle de soi, ils ne sont pas tout à fait à la hauteur en ce qui a trait aux normes de la masculinité 
"En ce qui a trait à la masculinité dite "subordonnée ", Connell (1995) la décrit comme étant une position dominée. Ceux qui en font partie sont marginalisés et peuvent être soumis à des comportements excessifs ou injustes. Ils sont parfois persécutés..." idéalisée (Cornwall, et Lindisfarne, 1995). Malgré leurs tentatives d'interagir naturellement avec les garçons du groupe dominant et d'intégrer le groupe supérieur, ils sont tolérés, mais étiquetés de "wannabes " (Adler, et Adler, 1998) par le groupe dominant. Leurs fréquentations sont souvent inadéquates en raison de leur gaucherie ou de leur maladresse. Dans les faits, ils ne saisissent pas toujours les blagues, ne savent pas comment agir en présence des filles et ont peu de repères quant aux événements qui les entourent, que ce soit à l'école ou ailleurs. Même s'ils ne peuvent pas s'intégrer complètement au groupe hégémonique, les membres du groupe complice bénéficient d'une certaine approbation des dominants.

En ce qui a trait à la masculinité dite «subordonnée ", Connell (1995) la décrit comme étant une position dominée. Ceux qui en font partie sont marginalisés et peuvent être soumis à des comportements excessifs ou injustes. Ils sont parfois persécutés par des mesures faisant appel à la violence. Ces garçons sont également étiquetés comme étant faibles et inférieurs. Conséquemment, ils sont mis à l'écart lors des activités sociales et ils ont peu d'occasions d'observer d'autres jeunes et d'interagir avec eux. Bref, ils demeurent confinés dans leur rôle de subordonnés et ont peu de chances de développer leurs habiletés sociales à l'adolescence. Pour sa part, Skelton (2001) maintient que ceux qui correspondent à la masculinité hégémonique se définissent par rapport à la masculinité subordonnée afin d'assurer la survie de leurs normes. À cet égard, toutes les formes de masculinités se définissent en opposition à une conception traditionnelle et unidimensionnelle de la féminité; les masculinités qui se trouvent au bas de la hiérarchie sont donc considérées comme étant une forme ou une autre de féminité, c'est-à-dire différentes de la masculinité dominante (Gilbert, et Gilbert, 1998). Quant à Swain (2006), il propose qu'être différent symbolise l'infériorité et représente donc une situation peu enviable pour quiconque désire réussir son adolescence. Dans ce groupe, nous retrouvons des garçons qui sont en général obéissants, polis, passifs, immatures, ainsi que ceux ayant des traits physiques particuliers ou moins de résistance physique. Nous pouvons ajouter à cette liste, les garçons 
qui participent peu ou pas aux activités sportives ou aux jeux physiques, qui ne regardent pas certaines émissions télévision et qui ont un faible intérêt pour les sports.

\section{Cadre conceptuel}

"L'étude des

garçons affichant

des caractéristiques

propres à ces catégories

nous a permis de

mieux comprendre

l'interprétation

qu'ils donnaient à la masculinité et ainsi le sens qu'ils donnaient à la construction de leur masculinité..."
Pour analyser les données de notre étude, nous avons retenu deux théories : la théorie sociale du genre (Connell, 1987;1995;2002) avec approfondissement de l'aspect de la masculinité hégémonique et celle des communautés de pratique (Wenger, 2005). Ces théories nous semblent pertinentes, car elles intègrent une perspective sociale de l'apprentissage à la création de significations et d'identité.Aux fins de notre recherche, la création de significations et d'identité se réfêre au développement de la masculinité des garçons, et ce, dans diverses communautés de pratique et différents groupes sociaux présents en milieu scolaire.

D'abord, la construction de la masculinité des garçons à l'école provoque un questionnement intimement lié à la théorie sociale du genre et plus particulièrement à la masculinité hégémonique (la domination de la part du sexe masculin), à la masculinité complice (forme aspirante de la masculinité dominante) et à la masculinité subordonnée (masculinité qui se trouve dans une position dominée). L'étude des garçons affichant des caractéristiques propres à ces catégories nous a permis de mieux comprendre l'interprétation qu'ils donnaient à la masculinité et ainsi le sens qu'ils donnaient à la construction de leur masculinité. Connell $(1987 ; 1995 ; 2002)$ soutient que la forme hégémonique peut varier d'une école à une autre selon la culture du milieu et que celle-ci se mobilise souvent autour des concepts socioculturels tels que l'habileté athlétique, la compétitivité, le courage et la robustesse. Selon Connell (2008), l'établissement scolaire permet la construction de la masculinité, car il s'agit du milieu social dans lequel des centaines d'élèves se regroupent. Il s'agit là du principal lieu d'actualisation de la masculinité. Les élèves amorcent, rejettent, refusent et imposent des modes de comportements, des tendances et des adhésions 
"L'étude de

l'hégémonie nous a permis d'identifier la hiérarchie des différentes masculinités dans le milieu qui a fait l'objet de l'étude. »

\section{"Enfin, l'étude de} l'hégémonie nous a permis de constater si les garçons ressentent de la pression à se conformer à une masculinité afin de se tailler une place parmi les autres garçons de l'école. " liés à la masculinité. Connell (2008) soutient que dans un tel contexte, les relations entre les masculinités dominantes, les masculinités complices et les masculinités subordonnées seront une préoccupation et possiblement une source de turbulence en ce qui a trait à la construction de la masculinité des garçons.

L'étude de l'hégémonie nous a permis d'identifier la hiérarchie des différentes masculinités dans le milieu qui a fait l'objet de l'étude. De plus, nous avons pu en connaitre davantage sur les participants de notre étude en ce qui a trait à leur adhésion à la masculinité idéalisée et nous avons pu identifier les garçons qui détiennent le pouvoir dans ce milieu scolaire. Nous avons pu également nous informer sur l'importance qu'accordent les garçons aux caractéristiques, aux comportements et aux valeurs de la masculinité locale et, par ricochet, aux différences entre les masculinités de ce milieu scolaire. Enfin, l'étude de l'hégémonie nous a permis de constater si les garçons ressentent de la pression à se conformer à une masculinité afin de se tailler une place parmi les autres garçons de l'école.

Deuxièmement, en complément à la théorie sociale du genre de Connell (1987; 1995; 2002), la théorie des communautés de pratique (Wenger, 2005) est utile à l'étude de la construction de la masculinité. En effet, selon Paechter (2003), différentes masculinités se manifestent dans différentes situations sociales. En d'autres termes, les hommes et les garçons sont constamment en train de négocier des attitudes, des gestes, des idées, des idéologies ou des comportements associés aux diverses masculinités présentes dans les communautés de pratique de leur environnement afin de se tailler une place parmi les autres membres. Au moyen d'un engagement mutuel au sein d'une entreprise commune, il se crée un répertoire partagé au sein des participants. Ce répertoire permet aux garçons de juger ce qui est important ou moins important, ce qu'ils doivent ignorer, ce à quoi ils doivent prêter beaucoup d'attention, ce qu'ils doivent dire et ce qu'ils ne doivent pas dire. Bref, ils apprennent les subtilités ainsi que les complexités de la masculinité dans différents contextes sociaux. À l'école, celui qui réussit à bien comprendre les subtilités de la masculinité lors des interactions augmente ses chances de devenir membre 
"...étudier le

développement de la masculinité au sein

des communautés de pratique pourrait

être une stratégie qui s'avère fructueuse afin de pouvoir comprendre ce phénomène à la fois significatif et complexe." à part entière de la communauté de pratique (Wenger, 1998). En lien avec ce qui précède, Paechter (2003) soutient que bon nombre de garçons sont simultanément membres de différentes communautés de pratique et renégocient perpétuellement le sens des différentes masculinités dans chacune des communautés dont ils sont membres, comme lorsqu'ils font partie d'une équipe sportive, sont élèves d'un programme spécial et participent à diverses activités culturelles à l'école. Tout compte fait, Paechter (2003) maintient qu'étudier le développement de la masculinité au sein des communautés de pratique pourrait être une stratégie qui s'avère fructueuse afin de pouvoir comprendre ce phénomène à la fois significatif et complexe. La théorie des communautés de pratique démontre clairement que l'apprentissage informel se produit au sein du contexte d'une communauté de pratique et que les relations et les pratiques associées à cette dernière dégagent un sens et une identité dans l'enceinte de cette communauté de pratique spécifique.

\section{Échantillon}

Un échantillonnage de convenance a été constitué pour la présente étude. Le recrutement de participants de l'école devait assurer la confidentialité des renseignements fournis et l'anonymat des garçons qui ont participé aux entrevues. L'échantillon était composé de seize garçons de onzième et de douzième année (16 à 18 ans) de divers groupes sociaux et de diverses communautés de pratique présentes à l'école qui s'étaient portés volontaires. Il importe de noter que nous distinguons les communautés de pratique des groupes sociaux étudiés dans le cadre de notre étude, car notre analyse des données nous a permis de constater que certains groupes étudiés se rapprochent davantage de groupes sociaux que de communautés de pratique au sens théorique même du terme. Bref, nous n'avons pas toujours pu clairement discerner certains éléments clés de la théorie des communautés de pratique au sein des cinq groupes de garçons du milieu scolaire étudié lors de notre analyse. À cet égard, deux communautés de 
pratique ressortent distinctement, soit les jocks et les fêtards, alors que les élèves studieux, les artistes et les non-affiliés correspondent à des groupes sociaux.

\section{Procédure}

La première étape en ce qui a trait au recrutement des participants consistait à se rendre dans les salles de classe des garçons de la onzième année ( 5 classes) et de la douzième année (4 classes). Le but était de faire une brève présentation des objectifs de la recherche pour que les garçons souhaitant y participer sachent ce que le chercheur ferait des informations recueillies lors des entrevues semi-structurées. Ces renseignements ont été communiqués aux élèves lors des visites en salle de classe afin de rassurer les participants.

Nous avons invité tout garçon qui pourrait être intéressé à participer aux entrevues à communiquer avec le chercheur par courrier électronique ou par téléphone, et ce, afin d'assurer leur anonymat. À la suite de chaque présentation, le chercheur a communiqué ses coordonnées, la lettre de recrutement destinée aux parents, le formulaire d'assentiment pour mineurs et le formulaire de consentement parental à tous les garçons afin que ceux qui veulent participer à l'entrevue puissent en discuter avec leurs parents et par la suite communiquer avec le chercheur.

Nous avons aussi communiqué avec les parents ou tuteurs des participants afin d'obtenir leur autorisation écrite. Enfin, comme énoncé précédemment, en raison des considérations éthiques en lien avec le recrutement des participants, nous devions viser le volontariat absolu en ce qui a trait à leur recrutement.

\section{Instrument et regard rétrospectif}

Il importe de souligner que l'instrument d'entrevue était un bref schéma d'entretien comportant des questions, la plupart ouvertes, 
articulées essentiellement autour d'opinions, d'impressions ou de jugements divers reliés au développement de la masculinité en milieu scolaire. L'instrument d'entrevue a été inspiré du schéma d'entretien utilisé par Frosh, Phoenix et Pattman (2002) qui vise délibérément à permettre aux participants d'exprimer leurs idées et leurs perceptions concernant la masculinité et portant, entre autres, sur leurs intérêts, la popularité des garçons, leurs rapports avec les filles, les différences entre les filles et les garçons. Voici quelques exemples de questions posées au cours des entrevues : "Quelles compétences sociales doit avoir un garçon pour être populaire. Possèdes-tu ces qualités? "; « Pourrais-tu me décrire les différents groupes d'amis que tu observes à ton école? "; «Quels sont les comportements des garçons au sein de ces différents groupes? ॥; « Existe-t-il différents types de filles? De gars? »

Notre connaissance du milieu scolaire acquise au cours de nos trois années d'expérience en tant qu'enseignant et conseiller en orientation nous a permis de présenter un regard rétrospectif sur le sujet à l'étude et de valider plusieurs constats présentés par les participants lors des entrevues. Par le biais de nos nombreuses observations, de nos échanges fréquents avec les élèves et d'un entretien avec un ancien collègue membre de l'administration, notre réflexion sur la construction de la masculinité des garçons de cette école nous a permis de présenter des informations sur le sujet à l'étude et de mieux interpréter les données collectées lors des entrevues.

\section{Résultats}

La présence de groupes sociaux et des communautés de pratique a émergé lors des entretiens avec les participants. Effectivement, ces derniers ont identifié des groupes sociaux et des communautés de pratique importantes dans leur milieu scolaire. Ainsi, nous avons analysé deux communautés de pratique soit celles des jocks et des fêtards, et trois groupes sociaux, c'est-à-dire les élèves studieux, les flotteurs et les artistes, en lien avec la masculinité en profondeur. 
En premier lieu, la communauté de pratique des jocks comprend les fervents d'activités sportives. L'engagement mutuel des jocks fait en sorte qu'ils consacrent beaucoup de temps aux sports. Le fait d'être membre des jocks établit un certain type de relation entre ces garçons et repose sur leur intérêt passionné pour le sport, sur les objectifs qu'ils se fixent tels que des victoires et championnats ainsi que sur une appartenance à un regroupement de jeunes qui partagent l'idée selon laquelle les "vrais hommes " font du sport. La participation aux sports semble avoir une influence sur la socialisation des participants en ce qui a trait au développement de leur masculinité. Les résultats de notre analyse corroborent ceux des chercheurs Foley (2001), Messner (2002), Steinfeldt, et collab. (2009) et Whannel (2007). En ce sens, le garçon ressent une pression de performer à un haut niveau et de se conformer à la notion selon laquelle un jock affiche des caractéristiques dans son répertoire partagé telles que la robustesse, l'habileté athlétique et un physique imposant tel qu'énoncé par ce garçon :

"Oui [les jocks font] juste des sports. Ils sont un peu plus grands que les autres. La taille physique et le sport. » (Garçon B04)

Selon plusieurs chercheurs, les garçons qui possèdent ces caractéristiques et ces habiletés sont souvent reconnus comme ayant des «traits de la masculinité » (Connolly, 1994; Mosse, 1996; Renold, 2005; Martino, 1999; Swain, 2002; Frosh, Phoenix et Pattmann, 2002). Notre regard rétrospectif qui comprend trois ans d'observation dans le milieu scolaire étudié ainsi que nos données des entrevues semi-structurées nous permettent de constater que ces caractéristiques semblent importantes pour les garçons, car elles leur permettent de s'afficher comme étant athlétiques et de se démarquer des autres garçons. Ce genre de ségrégation qui consiste à se distancier de tout garçon qui ne semble pas posséder les attributs des jocks est appuyé par les chercheurs Hauge et Haavind (2011) qui soutiennent que ces garçons se considéreront comme plus forts, plus athlétiques et plus robustes que les autres garçons de leur entourage. Selon le sport, certaines 
"...certaines

caractéristiques

sont valorisées par

les coéquipiers, les

entraîneurs et les

spectateurs, ce qui

contribue davantage à

la popularité du garçon

doué en sport et ainsi

à l'idée qu'un "vrai » garçon fait du sport.

Seulement certains

garçons semblent

réussir à faire partie de

ce groupe exclusif..." caractéristiques sont valorisées par les coéquipiers, les entraîneurs et les spectateurs, ce qui contribue davantage à la popularité du garçon doué en sport et ainsi à l'idée qu'un «vrai " garçon fait du sport. Seulement certains garçons semblent réussir à faire partie de ce groupe exclusif comme l'indique le commentaire suivant :

"Tout le temps parce qu'ils sont plus sportifs, plus socials, ils sortent tout le temps, ils sont jamais tout seuls, ils ont toujours été comme ça. C'est difficile de devenir populaire. Moi j'avais l'habitude d'être comme gêné, mais là je suis pas comme super bon comme eux. Le garçon populaire, il faut qui sache qu'est-ce qu'i[1] fait dans un sport. »(Garçon D04)

Ce qui semble unifier les jocks, c'est-à-dire leur entreprise commune, c'est leur passion ainsi que leur succès dans le domaine des sports. La grande majorité des participants réifie l'adhésion que les hommes aiment généralement les sports, qu'ils s'y adonnent en grand nombre et que l'habileté athlétique est intimement liée à la masculinité. En plus, d'autres éléments de réification tels que la popularité reconnue et la notion de pouvoir que les jocks détiennent face aux autres groupes sociaux leur permettent d'afficher leur masculinité et contribuent également à leur croyance de supériorité masculine. Les témoignages qui suivent soutiennent cette idée :

«C'est certain que les sportifs vont se penser comme la gang de populaires ou de... avec leur tête haute et leur ego super gros. Ils demandent beaucoup d'attention. »(Garçon A04)

«J'pense que ça affaire avec les sports, les affaires qu'ils font... La plupart des gars populaires, ils sont bons dans le sport, ils jouent toutes les sports. »(Garçon C02)

«Les jocks sont vraiment en forme. Ils se tiennent plus haut. Tu vois qu'ils ont de la confiance. » (Garçon A03) 
"...il nous semble que les membres de cette communauté de pratique occupent le rang le plus élevé dans la hiérarchie masculine des participants de notre recherche. En fin de compte, ils semblent servir de modèles masculins pour bon nombre d'entre eux. "
Nixon (1992) corrobore nos propos ainsi que ceux des participants et postule que les garçons qui connaissent du succès dans le domaine du sport et qui s'identifient au rôle d'athlète se distinguent souvent des autres garçons qui n'en font pas partie.

En outre, les jocks présentent des caractéristiques liées à la masculinité hégémonique. Nous réitérons que leur répertoire partagé comprend des traits typiques et traditionnels de l'homme idéal, dont l'habileté athlétique, la robustesse et un corps attrayant. Les jocks bénéficient d'un prestige auprès des jeunes de leur entourage et se retrouvent au sommet de la hiérarchie de la masculinité en milieu scolaire. Les jocks amorcent leur négociation de la masculinité en se référant à ces caractéristiques typiquement considérées comme étant masculines et considèrent les autres garçons, c'est-à-dire, ceux du milieu scolaire qui ne participent pas aux activités sportives, comme ayant moins de pouvoir, comme étant moins populaires et ainsi moins masculins :

"Quand tu es moins populaire, c'est plutôt si tu es un peu réservé, tu fais pas partie des équipes, des groupes, alors c'est plus que le monde te connait pas. » (Garçon A01)

« Ceux qui sont moins populaires, plus leurs intérêts, qu'est-ce qui font vraiment, au lieu de jouer les sports, ils vont jouer aux vidéogames, ils vont pas sortir aussi souvent, ils vont rester à la maison. » (Garçon B02)

Enfin, il nous semble que les membres de cette communauté de pratique occupent le rang le plus élevé dans la hiérarchie masculine des participants de notre recherche. En fin de compte, ils semblent servir de modèles masculins pour bon nombre d'entre eux.

En deuxième lieu, nous retrouvons les membres de la communauté de pratique des fêtards. Selon nos participants, ils consomment cigarettes, drogues et alcool, en bref, ce sont des fêtards : 
«Ceux qui vont aux parties, qui sont invités aux parties, ceux qui vont se faire en trouble. Ils vont boire, sex, drugs, whatever.» (Garçon C03)

Les fêtards n'adhèrent pas nécessairement à la culture du milieu scolaire étudié, où les activités sportives et les attentes au niveau de la réussite scolaire semblent être importantes pour la majorité de nos participants. Les sports et les exigences académiques nécessitent un engagement et un dévouement de la part des garçons. Malgré le fait que certains fêtards semblent participer sporadiquement aux activités sportives et suivent des cours à l'école, leur engagement mutuel relève beaucoup plus d'activités de fêtards. Bref, les règlements et les attentes du milieu scolaire ne semblent pas contenter les fêtards et leur identité rebelle. Ils semblent se conformer plutôt à des comportements considérés comme adultes et ils croient ainsi projeter une image de maturité auprès de leurs pairs. Tout comme les jocks, leur répertoire partagé comprend des comportements jugés comme étant masculins par nos participants, c'est-à-dire des comportements tels que

"Ils semblent vouloir réifier l'image d'une personne indépendante et mature en raison de leur comportement de fauteurs de trouble et, tout comme les jocks, nous considérons qu'ils sont au rang élevé de l'ordre hiérarchique du milieu scolaire étudié par rapport à la masculinité. Les fêtards ne se disent pas des plus engagés dans leurs études. Ils se montrent désintéressés..." l'indépendance, l'autonomie et la défiance. Ils semblent vouloir réifier l'image d'une personne indépendante et mature en raison de leur comportement de fauteurs de trouble et, tout comme les jocks, nous considérons qu'ils sont au rang élevé de l'ordre hiérarchique du milieu scolaire étudié par rapport à la masculinité. Les fêtards ne se disent pas des plus engagés dans leurs études. Ils se montrent désintéressés, sèchent souvent des cours et disent ne pas vouloir se fixer des buts précis en ce qui a trait à leur cheminement scolaire. Ils disent se concentrer plutôt sur leur vie sociale :

«Et puis comme des fêtards, everything, c'est plus like laid back, don't really care, ils vont pas en classe.» (Garçon B02)

L'entreprise commune des fêtards se rapporte à une réputation de consommateurs de drogues et d'alcool et à d'autres activités qui les amènent à défier l'autorité que représente l'administration de l'école. Ces comportements unifient ces garçons et reflètent leur conception masculine qui, elle, est fondée sur le rejet du conformisme. 
«En troisième lieu, nous examinons le groupe social des élèves studieux. Les membres de ce groupe disent se consacrer à leurs études. Ils semblent être à l'écart de tout autre groupe en milieu scolaire et ne jouissent pas d'une réputation reluisante auprès des autres garçons sur le plan social."
"Il faut que tu connais les règles pour les casser. Eux autres, ils vont casser les règles, ils vont toujours regarder comme le bad guy ou le bad guy, whatever. En faisant ça, tout le monde regarde. Eux autres sont cool, ils sont populaires." (Garçon C03)

Conséquemment, leur engagement à cette conception de la masculinité semble réifier une notion d'indépendance et d'autonomie. Quelques participants nous ont dit qu'un vrai homme prend ses propres décisions et ne se laisse pas influencer par d'autres. Ceux qui adhèrent incontestablement à cette représentation de la masculinité peuvent être membres de la communauté de pratique des fêtards. Tenant compte de la théorie sociale de Connell (1987; 1995; 2002), les fêtards peuvent être identifiés à l'image des durs et des fauteurs de trouble, des traits reliés à la masculinité hégémonique. Conséquemment, ils semblent exercer un pouvoir sur d'autres garçons de leur milieu et occupent ainsi un rang élevé dans la hiérarchie masculine du terrain étudié.

En troisième lieu, nous examinons le groupe social des élèves studieux. Les membres de ce groupe disent se consacrer à leurs études. Ils semblent être à l'écart de tout autre groupe en milieu scolaire et ne jouissent pas d'une réputation reluisante auprès des autres garçons sur le plan social. L'image réifiée est celle d'un garçon qui a du mal à s'intégrer à d'autres groupes sociaux et qui s'adapte difficilement à différentes situations sociales.

«Un peu, comme les gars dans le BI (baccalauréat international). Ils sont kind of hidden. C'est vraiment tout. Personne les voit, personne a la chance d'interagir avec eux. Ils sont jamais là.» (Garçon B03)

Selon la majorité de nos participants et d'après nos observations personnelles en tant qu'enseignant et conseiller scolaire, les élèves studieux de cette école semblent isolés et se retrouver dans une position subordonnée en raison du fait qu'ils ne possèdent pas dans leur répertoire les caractéristiques masculines jugées comme étant traditionnelles dans ce milieu, telles que la force, l'habileté 
athlétique, un sens de l'humour spontané et la sociabilité. Par conséquent, toujours selon plusieurs de nos participants, les élèves studieux n'interagissent pas beaucoup avec les autres élèves à l'extérieur de leur groupe social.

"Oui, ils sont gênés ou ils prennent toute leur temps à faire leurs devoirs. Normalement du monde du BI [baccalauréat international], ils peuvent être populaires, mais c'est pas que le monde l'aime pas, c'est juste qu'ils sont toujours en train de faire leurs devoirs, ils sont comme diehard. Ils sortent pas vraiment souvent. " (Garçon D04)

"Oui, ils sortent pas beaucoup, ils étudient beaucoup, ils fait tous leurs devoirs I guess. Ils travaillent beaucoup, mais sortent pas la fin de semaine. » (Garçon D03)

"Ils semblent toutefois autonomes, matures et responsables vis-àvis de leurs objectifs scolaires. Ils consacrent plutôt leur énergie à leurs études et fréquentent d'autres élèves qui obtiennent de bons résultats scolaires. »
Ils semblent toutefois autonomes, matures et responsables visà-vis de leurs objectifs scolaires. Ils consacrent plutôt leur énergie à leurs études et fréquentent d'autres élèves qui obtiennent de bons résultats scolaires. L'entreprise commune des garçons studieux se réfere plutôt à des buts qui leur permettront d'accéder à des études supérieures où ils poursuivront des études postsecondaires dans un domaine particulier pour enfin exercer une profession de leur choix. Les éléments unificateurs du groupe des studieux semblent être leur intérêt pour les cours, la réussite scolaire et leur incapacité de s'intégrer aux autres activités sociales à l'extérieur de leur propre groupe. Par conséquent, ils sont considérés comme étant un groupe subordonné au sein de ce milieu.

La quatrième catégorie regroupée au sein de nos participants consiste dans ce que nous appelons les "non-affiliés", dans leurs propres termes les "loners" ou "losers ". Quoique nous ayons inclus les flotteurs parmi un des groupes sociaux identifiés par nos participants, il importe de noter que les non-affiliés semblent perçus plutôt comme étant solitaires et ne semblent pas appartenir un groupe comme tel. Les flotteurs ne semblent pas participer 
collectivement aux diverses activités en milieu scolaire. Les participants nous ont dit que tout comme les garçons studieux, les flotteurs ne possèdent pas dans leur répertoire les attributs typiques de la masculinité idéalisée de cette école et sont subséquemment relégués aux personnes subordonnées sur l'échelle de la masculinité de ce milieu scolaire. En effet, ils sont à l'écart de la plupart des groupes à l'école et passent inaperçus. Ayant peu d'occasions de découvrir, de cultiver ou de déployer leurs talents à l'école, les non-affiliés s'adonnent à très peu d'activités sociales, sportives ou autres. Selon les participants, l'image réifiée des flotteurs est celle de personnes réservées, passives et isolées. En d'autres mots, l'entreprise commune de ces garçons se rapporte à une incapacité de s'intégrer aux autres groupes sociaux.

"[Les non-affiliés] sont plus renfermés sur eux-mêmes, ils vont à l'école, c'est tout qu'ils font, ils essayent de devenir populaires des fois, mais ça fonctionne jamais. Ça fonctionne pas vraiment parce qu'ils veulent pas l'accepter. " (Garçon C03)

Les caractéristiques ci-dessus ne sont pas des traits habituellement relevés par les garçons de l'étude en rapport à la masculinité dominante et conséquemment, ces non-affiliés semblent marginalisés et se trouvent au bas de la hiérarchie masculine du milieu scolaire étudié. Le garçon perçu comme étant non affilié est à la fois une forme aspirante de la masculinité dominante et une forme dominée.

Le cinquième et dernier regroupement qui se dégage des données recueillies auprès de nos participants concerne les artistes qui, comme on peut le deviner, sont liés par un intérêt commun pour les arts. Ces garçons participent à l'harmonie, au jazz, à l'improvisation et au théâtre. Les artistes ne semblent pas jouir d'une grande popularité auprès de la majorité des garçons et l'image réifiée est celle des garçons qui sont considérés comme étant différents. Ils ne semblent pas disposer dans leur répertoire des traits valorisés par la masculinité dominante du milieu et, par conséquent, ne correspondent pas aux exigences hégémoniques et 
"...nous interprétons que la majorité des participants sont largement influencés par les comportements, les normes et les valeurs en lien avec la masculinité dominante de cette école..." à la forme masculine idéalisée des participants de notre recherche. Les artistes de notre milieu de recherche semblent confinés au rang des subordonnés à l'école. Deux participants ont révélé qu'ils se tenaient le plus loin possible du monde des arts et plusieurs garçons du milieu jugeaient de façon négative les artistes par rapport aux caractéristiques dominantes de la masculinité. Il existe parmi quelques participants une idée préconçue selon laquelle les artistes affichent des caractéristiques féminines et ils veulent donc éviter d'y être associés. D'ailleurs, certains participants ont déclaré qu'ils démontraient autrefois un intérêt pour les arts et ont abandonné leurs activités artistiques en raison du malaise manifesté auprès de leur entourage.

Notre étude a révélé que l'image de la masculinité réifiée par plusieurs de nos participants comprenait des éléments d'hégémonie. Sur seize participants, seulement quelques garçons n'ont pas tenu le même discours que la majorité en ce qui a trait à la représentation de la masculinité. Celle-ci était fondée sur des caractéristiques particulières pour décrire de "vrais hommes". Sur ce, nous interprétons que la majorité des participants sont largement influencés par les comportements, les normes et les valeurs en lien avec la masculinité dominante de cette école. Connell (2000) maintient qu'il y a une hiérarchie des masculinités et une ascendance par laquelle une forme de masculinité domine les autres et les masculinités des jocks et des fêtards semblent celles qui sont idéalisées à cette école. Les témoignages suivants illustrent bien les normes et les valeurs des groupes dominants de cette école en lien avec la masculinité :

«C'est une personnalité forte comparé aux filles, une voix un peu plus forte que les filles, c'est un peu plus grand que les filles, pas aussi timide que les filles, toujours prêt à jouer les sports, un peu plus impulsif, les gars sont plus impulsifs que les filles. (Garçon B04)

Un homme, quelqu'un qui a la confiance, qui est brave, qui est macho. Il se laisse pas faire. Quelqu'un peut pas vraiment l'attaquer émotionnellement. Il ne se laisse pas intimider. Il a toujours les 
"...les participants

de notre étude ont relevé plusieurs traits qui caractérisent la masculinité : l'habileté athlétique; la force, la robustesse; l'autonomie, la maturité, un sens de responsabilité; le savoir-faire; un sens de l'humour vif; la sociabilité; un physique attrayant; et les relations amoureuses avec les filles. " épaules hautes. Il a une bonne posture. Il est toujours en contrôle. Tu peux voir qu'il est une personnalité vraiment forte. Ça aide quand un homme parle fort. Des fois la façon qu'il s'habille, il démontre qu'il est un homme de haute classe, de vraiment important. Il faut qu'il soit brave. Il a pas peur. " (Garçon A03)

Selon nos participants, le répertoire partagé d'un vrai homme comprend, entre autres, l'habileté sportive, la confiance, la robustesse, le sens de l'humour et un sens du contrôle. Ces caractéristiques sont, selon la plupart de nos participants, l'apanage des hommes. L'analyse de nos données nous permet de déduire que plusieurs de nos participants semblent faire référence à la notion selon laquelle les vrais hommes sont unis par une entreprise commune qui a comme objectif de se distinguer de tout ce qui est perçu comme étant féminin et ainsi affirmer un pouvoir sur le sexe opposé. Cette notion est soutenue par les chercheurs Branscombe, et collab. (1999), Jetten et Spears (2003) et O’Neil (2008). Selon ces derniers, les garçons attribuent des caractéristiques distinctives et stéréotypées à un groupe en particulier. Si des éléments viennent menacer la particularité du groupe et de ses membres, la motivation à vouloir stéréotyper sera d'autant plus forte. Autrement dit, les garçons doivent posséder les caractéristiques énumérées ci-dessus. Les filles quant à elles, ne possèdent pas les mêmes caractéristiques. Au contraire, certains de nos participants les qualifient de fragiles, d'émotives et de sensibles. Cette attitude à l'égard des filles semble aussi fondée sur l'hégémonie et assure à ceux qui possèdent les caractéristiques proprement masculines un certain pouvoir sur les filles ainsi que sur les garçons perçus comme possédant moins de traits valorisés par la masculinité dominante de ce milieu. Les réponses des participants portant sur les caractéristiques et particularités de la masculinité nous ont permis d'en dresser le portrait et ainsi d'interpréter la hiérarchie masculine des divers groupes de garçons qui semblent exister dans cette école. Pour réitérer, les participants de notre étude ont relevé plusieurs traits qui caractérisent la masculinité : l'habileté athlétique; la force, la robustesse; l'autonomie, la maturité, un sens de responsabilité; le savoir-faire; un sens de l'humour vif; la sociabilité; un physique attrayant; et les relations amoureuses avec les filles. 
"Tout compte fait, notre analyse des commentaires des garçons permet de brosser une représentation traditionnelle de la masculinité."
Tout compte fait, notre analyse des commentaires des garçons permet de brosser une représentation traditionnelle de la masculinité. Malgré une légère ouverture de la part des participants en ce qui concerne différents types de garçons et d'hommes, ils cherchent à développer les caractéristiques susmentionnées afin de s'éloigner du "féminin » et ainsi de s'affirmer dans leur masculinité. Ils considèrent les garçons moins populaires comme étant réservés, timides, asociaux et moins performants dans les sports. Les moins populaires disposent d'un réseau social limité ou n'ont pas une attitude "macho " pour se démarquer en tant qu'hommes. Leur représentation de la masculinité reflète bien la structure de cette école, qui favorise la culture sportive. Par les sports, les garçons peuvent démontrer leurs caractéristiques masculines très valorisées par le milieu. L'école met en place de nombreuses occasions où les garçons peuvent mettre leurs traits en valeur et afficher leur masculinité. Ainsi, se forme dans cette école une identité masculine qui se fonde principalement sur la participation aux activités sportives.

\section{Discussion}

Notre analyse nous permet de saisir la représentation que donnent nos participants à la construction de leur masculinité en contexte scolaire. Cette représentation découle, entre autres, des interactions avec les amis, les pairs et d'autres intervenants du milieu scolaire, du décodage des règles sociales ainsi que des relations hiérarchiques en milieu scolaire. Bref, la conception de la masculinité des participants à notre étude semble être apprise de façon informelle à l'école. En effet, ces derniers nous ont communiqué des informations abondantes sur la construction de la masculinité en contexte scolaire, et ce, même s'ils n'avaient jamais abordé le sujet du développement de la masculinité de façon formelle, c'est-à-dire dans le cadre d'un cours.

Tout d'abord, cette masculinité semble fondée sur un modèle traditionnel et comprend chez plusieurs garçons interviewés une 
"...plusieurs garçons croient qu'ils doivent adhérer aux normes et aux valeurs rattachées par la masculinité hégémonique afin d'adopter des comportements qui leur permettent de démontrer leur virilité.» adhésion à une construction de la masculinité hégémonique. Kenway et Fitzclarence (1997) et Mills (2001) soutiennent que la masculinité hégémonique est à la fois traditionnelle et habituelle et que plusieurs garçons croient qu'ils doivent adhérer aux normes et aux valeurs rattachées par la masculinité hégémonique afin d'adopter des comportements qui leur permettent de démontrer leur virilité. Pour leur part,Thompson et Pleck (1986) confirment nos propos en décrivant leur conceptualisation des normes masculines. Ils soutiennent que les hommes adhèrent à trois normes distinctes, soit celle du Big Wheel qui préconise que l'homme doive être respecté par les autres, celle du Sturdy Oak and Give em Hell qui reflète que l'homme doit être physiquement fort, robuste et agressif s'il le faut et, enfin, celle de l'antiféminin No Sissy Stuff selon laquelle un homme ne doit pas s'adonner à des activités considérées comme étant féminines. À cet égard, de nombreux chercheurs s'entendent pour dire que des croyances en lien avec la construction de la masculinité sont instaurées et imposées dès la naissance du garçon, qui apprend qu'il doit satisfaire aux normes sociétales telles que la dominance, la robustesse et l'hétérosexualité et doit éviter d'être passif, faible et homosexuel (Brannon, 1976; Deaux, et Kite, 1987; Herek, 1987; Kite, 2001).

L'image de la masculinité idéalisée de la plupart des participants semble rehaussée lorsque ceux-ci développent et acquièrent des caractéristiques telles que l'habileté athlétique, la robustesse, le savoir-faire avec les filles ainsi qu'une opposition à tout ce qui est considéré comme féminin chez les garçons. Messerschmidt (2000) abonde dans le même sens en ce qui concerne l'opposition de nos participants à la féminité en postulant que les garçons utilisent la sexualité afin de valider la masculinité en contexte scolaire. Selon ce chercheur, ceux-ci s'expriment et se définissent comme étant hétérosexuels en dénigrant fréquemment l'homosexualité et en adoptant des pratiques hétérosexuelles telles que les sorties avec des filles. De plus, Holland, Ramazanoglu et Sharpe (1993) maintiennent que les garçons qui ne démontrent pas ou peu d'intérêt pour les filles et qui ne possèdent pas les compétences pour pouvoir entretenir des relations hétérosexuelles sont étiquetés comme étant moins ou pas masculins. 
"Nos participants ont repéré chez les jocks une confiance, voire un air supérieur par rapport aux autres garçons de leur entourage. Pour leur part, les fêtards affichent une attitude rebelle, de l'autonomie et de la sociabilité. »
Notre analyse nous permet de constater que les communautés de pratique des jocks et des fêtards se retrouvent au sommet de la hiérarchie de la masculinité de notre milieu de recherche et représentent la masculinité dominante. Selon les participants de notre étude, les garçons de ces communautés de pratique semblent faire valoir certaines caractéristiques et certains comportements liés à la masculinité afin d'être membres d'une des deux communautés de pratique dominante du milieu de notre recherche. Selon les garçons interviewés, les jocks possèdent une grande habileté athlétique, une robustesse, et un physique attrayant. Pour ce qui est de leurs comportements, ceux-ci comprennent la participation à de nombreuses activités sportives et sociales qui semblent leur permettre de gagner de la confiance parmi leurs pairs en raison de leurs interactions abondantes. Nos participants ont repéré chez les jocks une confiance, voire un air supérieur par rapport aux autres garçons de leur entourage. Pour leur part, les fêtards affichent une attitude rebelle, de l'autonomie et de la sociabilité. Nos participants ont également décrit les fêtards comme étant des batailleurs qui tentent de se montrer plus forts que les autres. Les garçons faisant partie des deux communautés de pratique des jocks et des fêtards semblent vouloir effectivement adopter des comportements qui démontrent leur virilité et qui consolident ainsi leur masculinité. Les participants ont repéré plusieurs exemples de comportements qui contribuent à leur représentation d'une masculinité hégémonique. Parmi ceux-ci, de vrais garçons sacrent, se bagarrent, font de la musculation et du sport. L'ensemble de ces caractéristiques et comportements semble unifier les garçons des communautés de pratique des jocks et des fêtards, ce qui leur permet de bénéficier d'une popularité étendue parmi leurs pairs et d'être perçus comme étant de vrais garçons, supérieurs et distincts de ceux qui ne font pas partie de ces communautés de pratique.

Au sein de leur communauté de pratique, les jocks et les fêtards réalisent un apprentissage informel dont l'aboutissement semble être l'identité masculine. Ceux qui réussissent à se forger une appartenance à l'intérieur d'un de ces groupes seront, semble-til, davantage perçus comme étant masculins. Conséquemment, 
"...la représentation de la masculinité dans cette école semble très traditionnelle. "

"...que des garçons du milieu de recherche qui affichent ces comportements pourraient subir de la pression à se conformer à la version de la masculinité idéalisée. » cette perception semble aussi exercer une certaine pression sur l'ensemble des garçons à se conformer à une image hégémonique et très figée de la masculinité. Bref, selon nos participants, un garçon semble devoir posséder certains comportements et caractéristiques liés à la masculinité et ne devrait pas en dévier.

En rapport avec ce qui précède, cette image de la masculinité ne semble pas permettre aux participants de contempler d'autres types de masculinité. Par conséquent, elle les empêche d'explorer et de souscrire à une perspective à la fois personnelle et globalisante de la construction de la masculinité, ce qui nous permet de confirmer notre hypothèse selon laquelle la représentation de la masculinité dans cette école semble très traditionnelle. On semble adopter la position selon laquelle il existe une seule manière de devenir un homme et que les autres façons et les autres comportements sont considérés comme étant moins masculins et qu'ils sont par conséquent moins acceptés ou tolérés. Sous ce rapport, nos participants nous ont fait part d'un grand nombre de comportements à éviter à l'école. Ceuxci semblent renforcer et valoriser encore une fois un type de masculinité laissant peu de place aux diverses caractéristiques masculines. Selon les participants, les garçons devraient éviter de danser, de chanter, de parler des émotions, de participer aux arts dramatiques, de porter certains vêtements, de pleurer, de visionner des films romantiques, de se toucher entre eux et de partager leurs sentiments. Ces comportements semblent réservés aux garçons considérés comme étant moins masculins et ces faux pas masculins semblent également suggérer que des garçons du milieu de recherche qui affichent ces comportements pourraient subir de la pression à se conformer à la version de la masculinité idéalisée. Dans leur étude portant sur la pertinence de la masculinité hégémonique par rapport à la construction de la masculinité des garçons, Phoenix et Frosh (2001) maintiennent que des pratiques associées à la masculinité hégémonique peuvent être persuasives et puissantes et peuvent réguler les comportements des garçons à l'école et conséquemment influencer la construction de leur masculinité. À titre d'exemple, deux participants ont indiqué qu'ils se sont retirés de certaines activités afin d'échapper aux 
taquineries qu'elles entrainaient. Un autre nous a dit qu'il est important de suivre les normes de la société en ce qui a trait aux comportements considérés masculins afin d'éviter la "misère ". On peut se demander si d'autres garçons du milieu scolaire en question évitent d'afficher des comportements ou de participer à des activités quelconques de peur de s'exposer à des railleries. Est-ce que d'autres garçons craignent l'isolement parmi leurs pairs s'ils ne souscrivent pas à la masculinité dominante du milieu scolaire en question? Quels autres comportements ne sont pas tolérés? Pour leur part, les garçons appartenant aux groupes des artistes, des studieux et des flotteurs sentent-ils tous les effets de la masculinité dominante qui semble s'imposer dans cette école en raison de leurs caractéristiques et de leurs comportements qui different de ceux de la masculinité dominante et quels en sont effets sur leur vécu scolaire?

Toujours selon l'idée que l'image de la masculinité de nos

"...quelques

participants n'ont

pas nécessairement

tenu le même discours

et ont exprimé une

ouverture par rapport

à l'équité entre les sexes à l'école... Cette tendance à en dire très peu donne l'impression d'un malaise chez certains participants à se prononcer sur la diversité de la masculinité et aller à l'encontre de la masculinité dominante du milieu en question. " articipants semble fondée sur un modèle traditionnel, lorsque nous décortiquons certaines de leurs réponses, celles-ci nous permettent de saisir que certains garçons semblent vouloir créer une distance entre eux et les filles. En d'autres mots, des attitudes misogynes à l'égard des filles semblent exister chez certains d'entre eux et sont une composante de leur représentation de la masculinité. Ce phénomène relève-t-il d'une culture misogyne qui serait présente dans ce milieu scolaire? Bon nombre de nos participants considèrent les filles comme étant des " princesses ", fragiles, émotives, sensibles et peu sportives. Ces idées reflètent le traditionalisme qui semble aussi régner autour de la conception de la masculinité adoptée par nos participants. Pour leur part, seulement quelques participants n'ont pas nécessairement tenu le même discours et ont exprimé une ouverture par rapport à l'équité entre les sexes à l'école, et ce, seulement à la suite d'une sollicitation de la part du chercheur. Cette tendance à en dire très peu donne l'impression d'un malaise chez certains participants à se prononcer sur la diversité de la masculinité et aller à l'encontre de la masculinité dominante du milieu en question. Il reste à savoir si ces garçons et d'autres garçons de l'école seraient plus aptes à approfondir et à enrichir leurs notions de la masculinité 
"...nous ne pouvons nous empêcher de nous demander si l'occasion se présente souvent officiellement à cette école d'apprendre, de réfléchir et d'échanger de façon formelle sur la masculinité, la féminité et les rôles sexuels des hommes et des femmes dans le but de reconnaître et d'apprécier leur évolution dans notre société." s'ils avaient l'occasion d'être encadrés par des intervenantes ou intervenants du milieu scolaire qui les amèneraient à explorer l'aspect multidimensionnel de l'homme.

Certains participants nous ont mentionné le fait qu'ils abordaient pour la première fois le sujet de la construction de la masculinité en raison de leur participation à notre projet de recherche. En conséquence, nous ne pouvons nous empêcher de nous demander si l'occasion se présente souvent officiellement à cette école d'apprendre, de réfléchir et d'échanger de façon formelle sur la masculinité, la féminité et les rôles sexuels des hommes et des femmes dans le but de reconnaître et d'apprécier leur évolution dans notre société. Il nous semble que cette lacune pourrait expliquer en partie les croyances traditionnelles sur la conception de la masculinité auxquelles adhèrent plusieurs participants à notre recherche. Si on ne leur donne pas l'occasion de prendre conscience qu'il existe différentes versions de la masculinité, ce qui conduit conséquemment à l'appréciation de ces dernières, ils risquent de conserver leurs croyances et leurs significations culturelles de la masculinité. Sur ce point, l'omission d'un enseignement sur la masculinité et la féminité semble avoir un effet sur la construction d'une masculinité traditionnelle et habituelle.

La représentation de la masculinité chez plusieurs de nos participants reposait sur certaines habiletés spécifiques, notamment sur l'habileté athlétique. Pour leur part, Edley et Wetherell (1997) ont interrogé 20 garçons de 15 à 18 ans et leurs résultats indiquent, parallèlement aux nôtres, que l'habileté athlétique et le sport sont des composantes significatives de la construction de la masculinité des garçons en contexte scolaire. Nous croyons que la culture du milieu scolaire en question mise beaucoup plus sur les activités sportives que les autres activités parascolaires et offre aux garçons de cette école une structure qui leur donne de nombreuses occasions de faire du sport.Ainsi, cette structure semble socialiser les garçons à adopter une version de la masculinité. Nos participants estiment que les athlètes sont très populaires à cette école et ils valorisent l'habileté sportive. Nous déduisons par conséquent que l'habileté athlétique est une composante fondamentale de la construction 
de leur masculinité. Cette représentation a été exprimée par plusieurs participants. Quant à la réussite scolaire, celle-ci ne semble pas pour autant nuire à la construction de leur masculinité. À l'exception de ceux appartenant à la communauté de pratique des fêtards, ils veulent généralement réussir à l'école et ne se considèrent pas comme étant moins masculins s'ils obtiennent de bons résultats dans leurs cours. Notamment, plusieurs de nos participants croient que la réussite académique leur permettra d'accéder aux professions et à un style de vie agréable. Selon plusieurs, les garçons qui réussissent bien leurs études seront bien rémunérés, ce qui confere de prestige notable par rapport à la masculinité. En d'autres termes, ce prestige social que confere l'argent est pour plusieurs un élément d'importance en ce qui concerne la construction de la masculinité et la représentation de ce qu'est un homme.

Malgré la coexistence de divers types de masculinités, plusieurs de nos participants ne semblent pas avoir évolué sur le plan de l'acceptation de la diversité masculine et préferent ne pas côtoyer certains garçons du milieu de recherche qui possèdent moins de caractéristiques masculines idéalisées. À cet égard, une ambivalence par rapport à la masculinité semble exister parmi certains participants. D'une part, ils reconnaissent que certains garçons dévient de la norme en ce qui a trait à la masculinité et, d'autre part, ils semblent tout de même ressentir un malaise face à cette diversité masculine de peur d'être considérés eux-mêmes

"...la conception de la masculinité semble incohérente chez plusieurs de nos participants et semble aller à l'encontre de l'acceptation, de la diversité et de l'inclusion, qui sont des concepts de base du système scolaire au Manitoba. » comme moins masculins. Bref, la conception de la masculinité semble incohérente chez plusieurs de nos participants et semble aller à l'encontre de l'acceptation, de la diversité et de l'inclusion, qui sont des concepts de base du système scolaire au Manitoba. L'idée de paraître moins masculin que les garçons dominants du milieu scolaire étudié semble angoissante, car certains de nos participants évitent de s'exposer à cette situation qui leur paraît à la fois inconfortable et défavorable. Ce genre de comportement est soutenu par les études de Branscombe, et collab. (1999) et celles de Falomir-Pichastro et Mugny (2009).

Nous constatons qu'il existe parfois un manque d'ouverture face aux différentes représentations de la masculinité. La culture 
"...la masculinité des garçons deviendrait possiblement plus diversifiée. » masculine dominante du terrain étudié met en valeur des traits et des comportements plutôt associés à la masculinité hégémonique et par conséquent, nous nous demandons quelles seront les conséquences liées à l'adhésion à cette masculinité idéalisée. Nous sommes convaincus que les intervenantes et intervenants scolaires, y compris le personnel enseignant, les travailleuses et travailleurs sociaux, les psychologues scolaires ainsi que les élèves de l'école en question bénéficieraient de la diffusion des résultats de notre étude. On peut se demander si une telle conception de la masculinité ainsi cultivée au sein de la gent masculine étudiante de leur école n'est pas exagérée et, si oui, si la situation ne devrait pas être examinée sous toutes ses coutures afin d'y remédier. Ces intervenantes ou intervenants pourraient considérer s'il n'y aurait pas lieu de promouvoir les autres activités parascolaires existantes et d'en introduire de nouvelles qui pourraient attirer l'adhésion de nouveaux adeptes et ainsi restaurer un certain équilibre et, au moins, atténuer la domination des activités sportives et athlétiques chez les garçons de l'école. Existerait-il des moyens à mettre en œuvre pour rendre d'autres activités (artistiques et scientifiques, par exemple) assez attrayantes pour rivaliser avec les activités sportives et athlétiques? Enfin, si ces efforts étaient mobilisés dans le cadre d'une stratégie concertée pour l'ensemble de l'école et appuyée par une majorité du personnel enseignant, il serait possible d'envisager qu'un équilibre plus sain pourrait en venir à s'installer sur le plan des activités parascolaires de l'école. Les activités sportives et athlétiques continueraient, bien sûr d'avoir droit de cité, mais leur statut pourrait être moins dominant et surtout, la masculinité des garçons deviendrait possiblement plus diversifiée.

\section{Limites de l'étude}

Il est indéniable que notre recherche comporte certaines limites. Cette dernière avait comme objectif de décrire et d'interpréter des tendances liées à la construction de la masculinité au sein des groupes sociaux et des communautés de pratique d'un milieu 
scolaire particulier. Pour ce faire, le profil recherché pour son échantillon était celui de garçons francophones de onzième et de douzième année, provenant de divers groupes sociaux et de diverses communautés de pratique présentes à l'école. Nous avons donc suscité le volontariat et convoqué à une entrevue ceux qui étaient intéressés à répondre à des questions portant sur la construction de la masculinité. Les garçons étaient entièrement libres d'y participer et l'entrevue était complètement indépendante du programme scolaire, n'étant d'aucune façon reliée aux cours ou aux évaluations. Quoique notre échantillon comptait des membres de divers regroupements du milieu de recherche, il aurait été bénéfique de pouvoir repérer un nombre plus ou moins égal de garçons provenant des trois programmes offerts à la même école (le programme régulier, le programme du baccalauréat international et le programme alternatif) afin de pouvoir déceler les différences liées à la construction de leur masculinité. Malheureusement, cela n'a pas été réalisé. Seul un petit nombre parmi la centaine de garçons a voulu participer à l'étude. Un autre élément qui n'a pas été examiné est celui de l'influence du niveau socioéconomique de chaque participant sur le développement de sa masculinité en contexte scolaire. Nous n'avons pas considéré la situation financière de la famille et son rôle sur la construction de la masculinité en contexte scolaire. De plus, à l'exception d'un garçon, tous les participants étaient canadiens et caucasiens. Le milieu de recherche compte pourtant de nombreux immigrants et la façon dont ces garçons négocient leur masculinité n'a pas été étudiée. Malgré cette omission, il importe de nous demander si nos résultats ont le potentiel d'être transférés aux autres garçons dont l'ethnie differe de celle des participants. Tel que nous l'avons déjà indiqué, de nombreux apprentissages informels ont lieu en milieu scolaire et les garçons apprennent les subtilités et les complexités de la masculinité dans divers contextes sociaux en milieu scolaire. Cette notion soutenue par Wenger postule que des compétences s'acquièrent surtout en marge des apprentissages scolaires. Ces compétences comprennent le décodage des règles sociales, les relations hiérarchiques en milieu scolaire et les règlements et conventions à l'extérieur ou à l'intérieur de la salle de classe. 
Bref, nous pensons que nos résultats peuvent être appliqués aux minorités de cette école, mais seule une étude plus poussée en lien avec la construction de la masculinité qui comprend toutes les ethnies de notre milieu de recherche pourrait le confirmer.

\section{Conclusion}

"...il s'avère important de se demander dans quelle mesure et de quelle manière ces éléments sont liés au contexte minoritaire francophone au Manitoba."
La présente étude nous a permis de mieux comprendre ce qu'est un garçon adolescent dans ce contexte d'une école francophone. Malgré l'évolution du concept de la masculinité et des rôles sexuels dans notre société au cours des dernières années, plusieurs de nos participants ne semblent pas accepter une grande diversité masculine. En effet, les adolescents que nous avons interviewés semblent s'accrocher à une perception simplifiée de la masculinité et vouloir construire la leur selon un modèle figé. Leur conception de la masculinité ne semble pas leur permettre d'en considérer d'autres versions. Leurs valeurs, croyances et attitudes donnent l'impression de correspondre à une masculinité traditionnelle et hégémonique qui est négociée et maintenue par l'entremise de leur participation ou de leur non-participation à des communautés de pratique particulières. À cet égard, il s'avère important de se demander dans quelle mesure et de quelle manière ces éléments sont liés au contexte minoritaire francophone au Manitoba. Par ailleurs, un manque d'idées différentes et novatrices par rapport à ce qu'est un homme et à la façon dont celui-ci construit sa masculinité semble contribuer à une rigidité qui pourrait les contraindre à cheminer en tant qu'homme en territoire sûr, sans sortir des sentiers battus. Par conséquent, quelles seront les conséquences liées à l'adhésion à cette masculinité idéalisée et quels pourraient être les impacts possibles pour les intervenantes et intervenants scolaires des écoles franco-manitobaines lorsqu'ils sont appelés à conjuguer avec ces élèves afin de les accompagner dans leur cheminement scolaire? Comme l'ont constaté plusieurs chercheurs (Blashill, et Wal, 2009; Harris, et Harper, 2008; Kierski, et Blazina, 2009; Peralta, 2007), l'adhésion à une masculinité 
hégémonique à l'adolescence peut entraîner dans la vie adulte des difficultés comme la dépression, l'abus d'alcool, divers problèmes de santé, des problèmes d'alimentation, des préoccupations extrêmes liées au corps parfait et l'incapacité de demander de l'aide. Il va sans dire que ces difficultés pourraient entrainer plus tard de grands défis pour les garçons et, par conséquent, pour la communauté franco-manitobaine dans son ensemble.

\section{Bibliographie}

ADLER, Particia, et Peter ADLER (1998). Peer power: Preadolescent culture and identity, Londres, Rutgers, $247 \mathrm{p}$.

ADLER, Patricia, Steven KLESS et Peter ADLER (1992). «Socializing to gender roles: Popularity among elementary school boys and girls ", Sociology of Education, Vol. 65, p. 169-187.

BEASLY, Christine (2008). « Rethinking hegemonic masculinity in a globalizing world ", Men and Masculinities, Vol. 11, p. 86-103.

BLASHILL, Aaron J., et Jillon S.WAL (2009). « Mediation of gender role conflict and eating Pathology in gay men ", Psychology of Men and Masculinity, Vol. 10, p. 204-217.

BRANNON, Robert (1976). "The male sex role: Our culture's blueprint for manhood, what it's done for us lately ", dans D. David et R. Brannon, The forty-nine percent majority: The male sex role, Reading, MA, Addison Wesley, p. 1-48.

BRANSCOME, Nyla R., et collab. (1999). "The context and content of social identity threats ", dans Naomi Ellemers, Russell Spears et Bertjan Doosje, Social identity: Context, commitment, content, Oxford, Blackwell, p. 35-38.

CARRIGAN, Tim, Raewyn W. CONNELL et John LEE. (1985). "Towards a new sociology of masculinity ", Theory and Society, Vol. 14, $\mathrm{N}^{\circ}$ 5, p. 551-602.

CONNELL, Raewyn W. (1987). Gender and power: Society, the person and sexual politics, Cambridge, Polity, $352 \mathrm{p}$.

CONNELL, Raewyn W. (1990). "An iron man: The body and some contradictions of hegemonic Masculinity ", dans M. A. Messner et D. F. Sabo, Sport, men and gender order: Critical feminist perspectives, Champaign, IL, Human Kinetics, p. 452-478.

CONNELL, Raewyn W. (1995). Masculinities, Cambridge, Polity, 295 p.

CONNELL, Raewyn W. (1996). "Teaching the boys: New research on masculinity and gender strategies for schools ", Teachers College Record, Vol. 98, № 2, p. 206-235.

CONNELL, Raewyn W. (2000). The men and the boys, Cambridge, Polity, $272 \mathrm{p}$.

CONNELL, Raewyn W. (2002). Gender Short Introductions, Cambridge, Polity, 184 p.

CONNELL, Raewyn W. (2008). «Masculinity construction and sports in boys' education: a framework for thinking about the issue ", Sport, Education and Society, Vol. 13, N 2, p. 131-145.

CONNELL, Raewyn W., et James W. MESSERSCHMIDT (2005). "Hegemonic masculinity: Rethinking the concept ", Gender and Society, Vol. 19, N 6, p. 829-859.

CONNOLLY, Paul (1994). "All lads together? Racism, masculinity and multicultural/anti-racist strategies in a primary school », International Studies in Sociology of Education, Vol. 4, N 2, p. 191-211. 
CORNWALL, Andrea, et Nancy LINDISFARNE (1995). Dislocating masculinity, Londres, Routledge, $234 \mathrm{p}$.

DEAUX, Kay, et Mary E. KITE (1987). «Thinking about gender », dans B. B. Hess et M. M. Feree, Analyzing gender: A handbook of social science research, Newbury Park, CA, Sage, p. 92-117.

EDLEY, Nigel, et Margaret WETHERELL (1997). " Jockeying for position: The construction of masculine Identities ", Discourse and Society, 8, p. 203-217.

FALOMIR-PICHASTOR, Juan, M., et Gabriel MUGNY (2009). «I'm not gay...I'm a real man! Heterosexual men's gender self-esteem and sexual prejudice ", Personality and Social Psychology Bulletin, 35, p. 1233-1243.

FOLEY, Douglas E. (2001). «The great American football ritual: Reproducing race, class, and gender in equality ", dans Andrew.Yiannakis et Merrill Melnick, Contemporary issues in sociology of sport, Champaign, IL, Human Kinetics, p. 23-40.

FRANK, Blye, et collab. (2003). "A tangle of trouble: boys, masculinity and schooling - future considerations ", Educational Review, Vol. 55, № 2, p. 119-133.

FROSH, Stephen, Ann PHOENIX et Rob PATTMAN (2002). Young masculinities, New York, Palgrave, $304 \mathrm{p}$.

GILBERT, Rob, et Pam GILBERT (1998). Masculinity goes to school, Londres, Routledge, 293 p.

HARRIS, Frank Harris III, et Shaun R. HARPER (2008). « Masculinities go to community college: Understanding male identity socialization and gender role conflict ", New Directions for Community Colleges, Vol. 142, p. 25-35.

HAUGE, Mona I., et Hanne HAAVIND (2011). «Boys' bodies and the constitution of adolescent masculinities ", Sport, Education and Society, Vol. 16, N¹, p. 1-16.

HEREK, Gregory. M. (1987). "On heterosexual masculinity: Some psychical consequences of the social construction of gender and sexuality ", The American Behavioral Scientist, 29, p. 563-577.

HOLLAND, Janet, Caroline RAMAZANOGLU et Sue SHARPE (1993). Wimp or gladiator: Contradictions in acquiring masculine sexuality, Londres, Tufnell, $40 \mathrm{p}$.

JETTEN, Jolanda, et Russell SPEARS (2003). «The divisive potential of differences and similarities: The role of intergroup distinctiveness in intergroup differentiation ", European Review of Social Psychology, Vol. 14, p. 203-241.

KEHLER, Michael D. (2004). "Masculinities and Resistance: High school boys (un)doing boy ", Taboo, Vol. 8, N 1, p. 97-113.

KENWAY, Jane, et Lindsay FITZCLARENCE (1997). " Masculinity, violence and schooling: Challenging poisonous pedagogies ", Gender and Education, Vol. 9, N 1, p. 117-133.

KIERSKI, Werner, et Christopher BLAZINA (2009). "The male fear of feminine and its effects on counseling and psychotherapy ", The Journal of Men's Studies, Vol. 17, p. 155-172.

KITE, Mary, E. (2001). " Changing times, changing gender roles: Who do we want women and men to be? ", dans R. Unger, The handbook of the psychology of women and gender, New York, Wiley, p. 215-227.

LEVANT, Ronald F, et collab. (2007). "Initial validation of the Male Role Norms InventoryRevised ", Journal of Men's Studies, Vol. 15, p. 83-100.

MANKOWSKI, Eric S., et Kenneth I. MATON (2010). «A community psychology of men and masculinity: Historical and conceptual review ", American Journal of Community Psychology, Vol. 45 , p. $73-86$. 
MARTINO, Wayne (1999). "Cool boys, party animals, squids and poofers : Interrogating the dynamics and politics of adolescent masculinities in school ", British Journal of Sociology of Education, Vol. 20, p. 239-263.

MESSERSCHMIDT, James W. (2000). Nine lives: Adolescent masculinities, the body, and violence, Boulder, CO, Westview, $168 \mathrm{p}$.

MESSNER, Michael A. (2002). Taking the field: Women, men and sports, Minneapolis, University of Minnesota Press, 280 p.

MESSNER, Michael A., et Donald SABO (1990). Sport, men and the gender order: Critical feminist Perspectives, Champaign, Il, Human Kinetics Books, 288 p.

MILLS, Martin (2001). Challenging violence in schools: An issue of masculinities, Buckingham, Open University, $165 \mathrm{p}$.

MOSSE, George L. (1996). The image of man: the creation of modern masculinity, Oxford, Oxford University Press, $232 \mathrm{p}$.

O'NEIL, James M. (2008). «Summarizing 25 years of research on men's gender role conflict using Gender Role Conflict Scale: New research paradigms and clinical implications ", The Counseling Psychologist, Vol. 36, p. 358-445.

PAECHTER, Carrie (2003). "Masculinities and femininities as communities of practice, Women's Studies International Forum, Vol. 26, No 1, p. 69-73.

PERALTA, Robert L. (2007). «College alcohol, use and the embodiment of hegemonic masculinity among European American men ", Sex Roles, Vol. 56, p. 741-756.

RENOLD, Emma (2005). Girls, boys and junior sexualities, Londres, Routledge Falmer, 206 p.

STEINFEDLT, Jesse A., et collab. (2009). "Gender role conflict and stigma toward help-seeking among college foot-ball players ", Psychology of Men and Masculinity, Vol. 10, p. 261-272.

SWAIN, Jon (2002). "The resources and strategies boys use to establish status in a junior school without competitive sport ", Discourse, Vol. 23, N 1, p. 91-107.

SWAIN, Jon (2005). "Masculinities in education ", dans Michael Kimmel, Jeff Hearn et Raewyn Connell, Handbook of Studies on Men and Masculinities, Thousand Oaks, CA, Sage, p. 213-229.

SWAIN, Jon (2006). «Reflections on patterns of masculinity in school settings », Men and Masculinities, Vol. 8, No 3, p. 331-349.

THOMPSON, Edward H., et Joseph H. PLECK (1986). "The structure of male role norms ", American Behavioral Scientist, Vol. 29, p. 531-543.

WENGER, Étienne (1998). Communities of practice. Learning, meaning and identity, New York, Cambridge University, $318 \mathrm{p}$.

WENGER, Etienne (2005). La théorie des communautés de pratique, traduction et adaptation de Fernand Gervais, Saint-Nicolas, QC, Les Presses de l'Université Laval, 309 p.

WETHERELL, Margaret, et Nigel EDLEY (1999). « Negotiating hegemonic masculinity: imaginary positions and psycho-discursive practices ", Feminism and Psychology, Vol. 9, N 3, p. 335-356.

WHANNEL, Garry (2007). " Mediating masculinities: The production of media representations in sport ", dans C. Carmichael Atkinson, Sport and gendered identities: Masculinities, femininities and sexualities, Londres, Routledge, p. 7-21. 